\title{
Free Space Optical Wireless Communications under Turbulence Channel Effect
}

\author{
Mazin Ali A. Ali \\ Department of Physics / College of Science / AL-Mustansiriyah Univ., Iraq-Baghdad.
}

\begin{abstract}
This article is focused on the turbulence attenuation and their effect on optical beam in free space. Analysis has been done for comparison the atmospheric turbulence effect between two classical methods, Rytov approximation and Andrews's method. The SNR was calculated for not weak turbulence employing Tailor series at different strength of scintillation. These calculations run for communications of wavelengths $850 \mathrm{~nm}$ and $1550 \mathrm{~nm}$. The distance between the transmitter and receiver o horizontal link set to value range (0-2000) $\mathrm{m}$.
\end{abstract}

Keywords: Rytov variance, Atmospheric turbulence, Rytov approximation, Andrews's method. Signal to noise ratio.

\section{Introduction}

In wireless optical communication links, atmospheric turbulence causes fluctuations in both the intensity and the phase of the received light signal, impairing link performance [1,2]. Turbulent fluctuations in the wind velocities in the upper atmosphere mix layers of differing temperatures, densities and water vapor content causes change in refractive index [1,2]. Thus the index of refraction of each level of the atmosphere fluctuates. The fluctuations originate from the energy conversion of solar energy. The solar power heats the atmosphere irregular, different cells in the atmosphere exhibit different temperatures and in the atmosphere appear turbulences. This reflects as variations in the index of refraction of the atmosphere that create fluctuations in the amplitude of the received optical signal with a frequency spectrum between 0.01 and $200 \mathrm{~Hz}$. This is due to the fact that light transmission in a medium occurs according to the principle that light traveling from one point to another follows the shortest optical path (Fermat's principle), and this depends not only on the geometrical distance, but also on the optical characteristics of the medium, from which one of the most important ones is the index of refraction. The index of refraction value in the atmosphere depends on temperature, pressure, and humidity of air and on the wavelength used for the transmission [3].

\section{Atmospheric Turbulence Model}

There are several models which describe atmospheric turbulence attenuation. There are the Rytov approximation and the Andrews's method. The Rytov method widely used to calculate the effect of the atmosphere turbulence. It gives quite good agreement with the experimental data [4]. The Andrews's method takes into account the size of receiver optical lens $D_{R E C}$.Not long ago it was published other method. It is called Method of Available Power [5].

\section{Andrews's Method}

This method is derived on the basis of a detailed mathematical analysis of the turbulence in atmospheric transmission media presented by Larry C. Andrews [6]. The resultant expression for the theoretical mean varianceof optical intensity is given as:

$$
-2 \sigma_{I}\left(D_{R E C}\right) \cong \exp \left[\begin{array}{c}
\frac{0.49 \beta_{0}^{2}}{\left(1+0.18 d^{2}+0.56 \beta_{0}^{12 / 5}\right)^{7 / 5}} \\
+\frac{0.51 \beta_{0}^{2}\left(1{ }_{-} 0.69 \beta_{0}^{12 / 5}\right)^{-5 / 6}}{1+0.9 d^{2}+0.62 d^{2} \cdot \beta_{0}^{12 / 5}}
\end{array}\right]-1(1)
$$

In relation (1), two parameters are used. The first parameter $\mathrm{d}$, which contains information about the wavelength of optical source, distance between the transmitter and receiver $\mathrm{L}$, and receiving optical lens diameter $\mathrm{D}_{\mathrm{REC}}$. 
$d=\sqrt{\frac{2 \pi}{4 \lambda L}} \cdot D_{R E C}$

While the second parameter is $\beta_{0}^{2}$ given by the relation

$\beta_{0}^{2}=0.5 C_{n}^{2} \cdot k^{7 / 6} L^{11 / 6}$

Where $C_{n}^{2}$, is the refractive index structure parameter, $\mathrm{k}$ is the wave number.

This method takes into account the size of receiving optical lens $D_{R E C}$. Aperture averaging is the most valuable asset of Andrews's method. Due to this fact we expect the lowest resultant turbulence attenuation when Andrews's method is applied. In this case the turbulence attenuation in $\mathrm{dB}$ is given by the relation [7]:

$\alpha_{\text {Andrews }}=\left|10 . \log \left(1-\sqrt{\frac{-2}{\sigma_{I}\left(D_{R E C}\right)}}\right)\right|$ (4)

\section{The Rytov Approximation Model}

The Rytov approximation starts from the premise that an air mass behaves as a fluid. On the basis of Rytov's analysis [6], the relationship between refractive index structure parameter $C_{n}^{2}$, which characterizes the volume of atmospheric turbulence and relative variance of optical intensity, $\sigma_{I, r e l}^{2}$ was set by Rytov as

$\sigma_{I, r e l}^{2}=K \cdot C_{n}^{2} \cdot k^{7 / 6} L^{11 / 6}$

Where $\mathrm{K}$ is the constant 1.23 for the plane wave and 0.5 for the spherical wave, this model sets scintillation variance $\sigma_{\chi}^{2}$ which is expressed by relation

$\sigma_{\chi}^{2}=23.17 C_{n}^{2} \cdot k^{7 / 6} \cdot L^{1116}$

The turbulence attenuation is related to scintillation and it is equal to $2 \sigma_{\chi}$ and thus the relation for turbulence attenuation $\alpha_{\text {Rytov }}$ can be written as [8]

$\alpha_{\text {Rytov }}=2 \cdot \sqrt{23 \cdot 17 \cdot C_{n}^{2} \cdot k^{7 / 6} \cdot L^{11 / 6}}$

This method is not concern with the effect of aperture averaging.

\section{Turbulence Effect And SNR Relation}

For a practical FSO system (plane wave) with weak and symmetrical atmosphere turbulence the log irradiance variance is given by [9]:

$\prec \chi^{2} \succ=0.31 C_{n}^{2} k^{7 / 6} L^{11 / 6}(8)$

Therefore, the noise source is the atmosphere turbulence

$\prec \chi \succ=\ln (1+\varepsilon)(9)$

$\varepsilon=A_{n}(r) / A_{0}(r)$

Where $\chi$ represent the fluctuations of the $\log$ of the amplitude of the field, $A_{n}(r)$ is the amplitude of noise, $\mathrm{A}_{0}(\mathrm{r})$ is the amplitude of laser beam $\mathrm{n}$ the atmosphere without turbulence.

The SNR in terms of mean signal and noise signal intensity $\mathrm{I}_{0}$ and $\left\langle\mathrm{I}_{n}\right\rangle$, respectively is given by[10]:

$S N R=\frac{I_{0}}{\prec I_{n} \succ}=\frac{\prec A_{0}^{2}(r) \succ}{\prec A_{n}^{2}(r) \succ}=\left[\prec \varepsilon^{2} \succ\right]^{-1}$ 
For weak turbulence mode, $\varepsilon$, is very small thus eq. (9) is given as [11]:

$\prec \chi \succ=\ln (1+\varepsilon) \approx \varepsilon(11)$

With this approximation SNR is written as

$S N R=\left[\prec \chi^{2} \succ\right]^{-1}=\left(0.31 C_{n}^{2} k^{7 / 6} L^{11 / 6}\right)^{-1}$

Without the above approximation eq. (11) is written as

$\prec \chi \succ=\ln (1+\varepsilon) \approx \varepsilon=\left(e^{\chi}-1\right)(13)$

For weak turbulence $\left(C_{n}^{2} \leq 10^{-14} m^{-2 / 3}\right)$, the SNR is given by eq. (12), when the turbulence is not weak $\left(C_{n}^{2} \geq 10^{-14} m^{-2 / 3}\right)$, the relationship between the SNR and $\chi$ is much more complicated. But by using the Tailor series for function $f(\chi)=\left(e^{\chi}-1\right)^{2}$ and with some simplification, eq. (10) can be written be approximated to [11]:

$$
S N R=\frac{1}{\prec \chi^{2}+\chi^{3}+\ldots \succ \succ \quad \alpha \prec \chi^{2} \succ}
$$

When $1 \leq \alpha \leq 2$ and represents the strength of scintillation (for worst case $\alpha=2$ )

\section{Results and discussions}

The calculation of turbulence attenuation carried out by matlab to show the effect of turbulence on free space optical communications. Atmospheric windows with wavelengths of $850 \mathrm{~nm}$ and $1550 \mathrm{~nm}$ are used in the computations. Refractive index structure parameters $C_{n}^{2}$ were set to $10^{-15} \mathrm{~m}^{-2 / 3}, 10^{-14} \mathrm{~m}^{-2 / 3}, 10^{-13} \mathrm{~m}^{-2 / 3}, 10^{-12} \mathrm{~m}^{-2 / 3}$. The first calculation (fig.1) proceeded comparing between the plane and spherical waves for the refractive index structure parameter $10^{-15} \mathrm{~m}^{-2 / 3}$. According to the results obtain the rytov approximation give different resultant attenuation for plane and spherical waves under wavelengths study $850 \mathrm{~nm}, 1550 \mathrm{~nm}$.

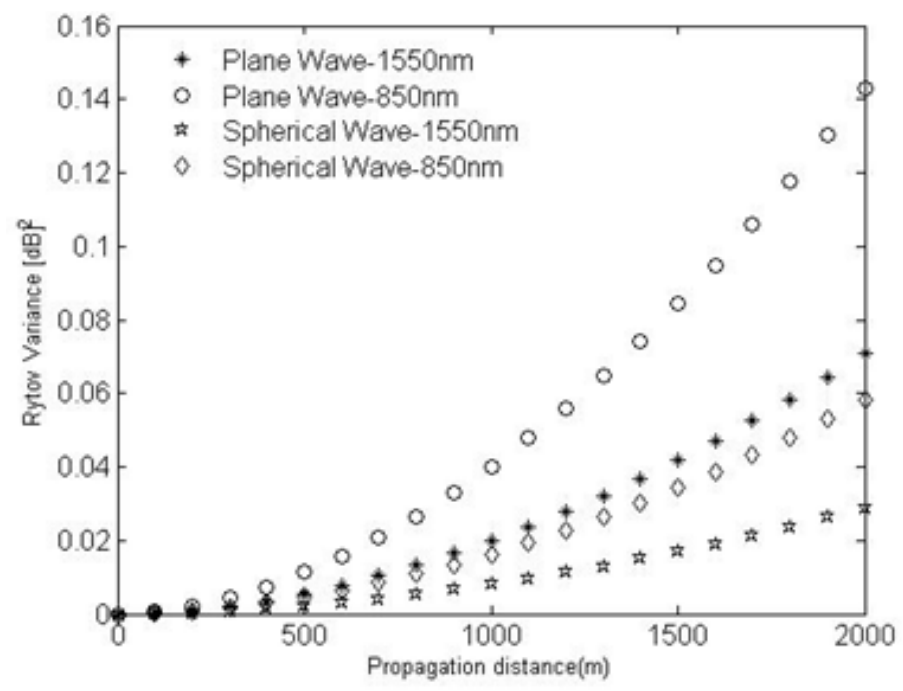

Fig. 1 Comparison of attenuation of the Relative variance for $C_{n}^{2}=10^{-15} \mathrm{~m}^{-23}$

The second calculation applied the refractive index structure parameter $10^{-14} \mathrm{~m}^{-2 / 3}$, which means a higher Rytov variance. It is evident that the Rytov variance increasing with increases the atmospheric turbulence. 


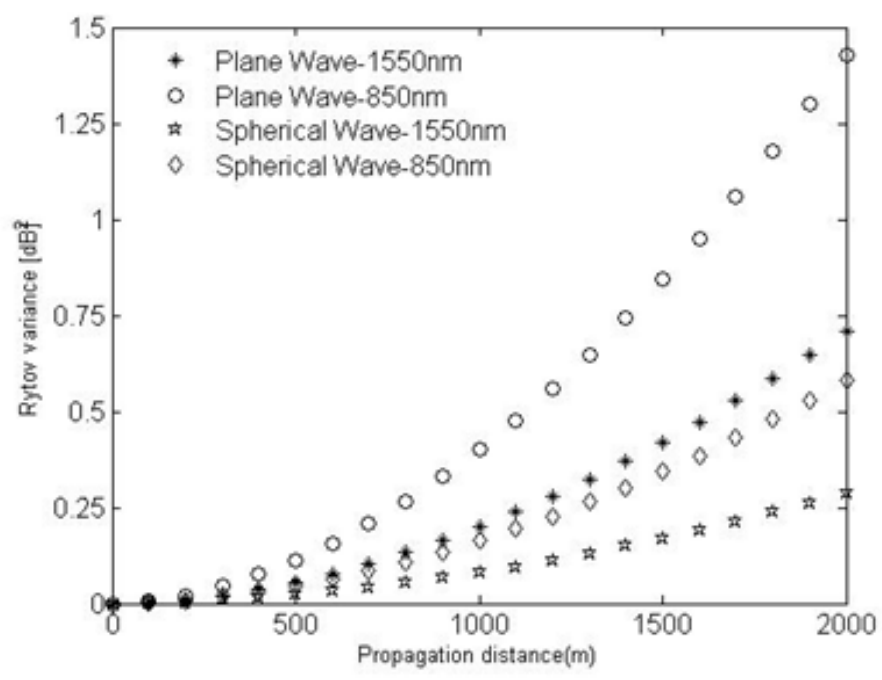

Fig. 2. Comparison of attenuation of the Relative variance for $C_{n}^{2}=10^{-14} \mathrm{~m}^{-23}$

When we calculate with $C_{n}^{2}=10^{-13} \mathrm{~m}^{-2 / 3}, 10^{-12} \mathrm{~m}^{-2 / 3}$ (higher atmospheric turbulence), the trend of turbulence attenuation is take the same behavior. We obtain characteristics as shown in fig. $(3,4)$. More over rytov approximation give different results for plane and spherical waves for wavelengths under study.

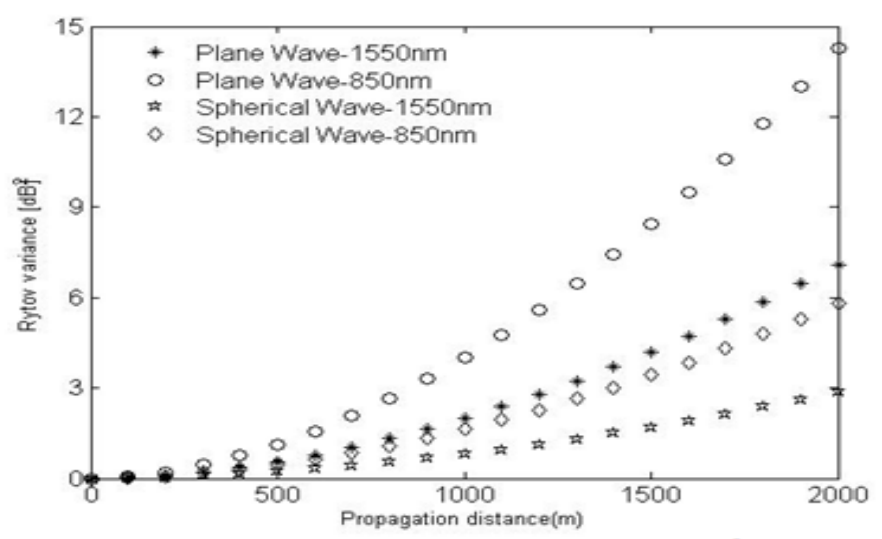

Fig. 3. Comparison of attenuation of the Relative variance for $C_{n}^{2}=10^{-13} \mathrm{~m}^{-23}$

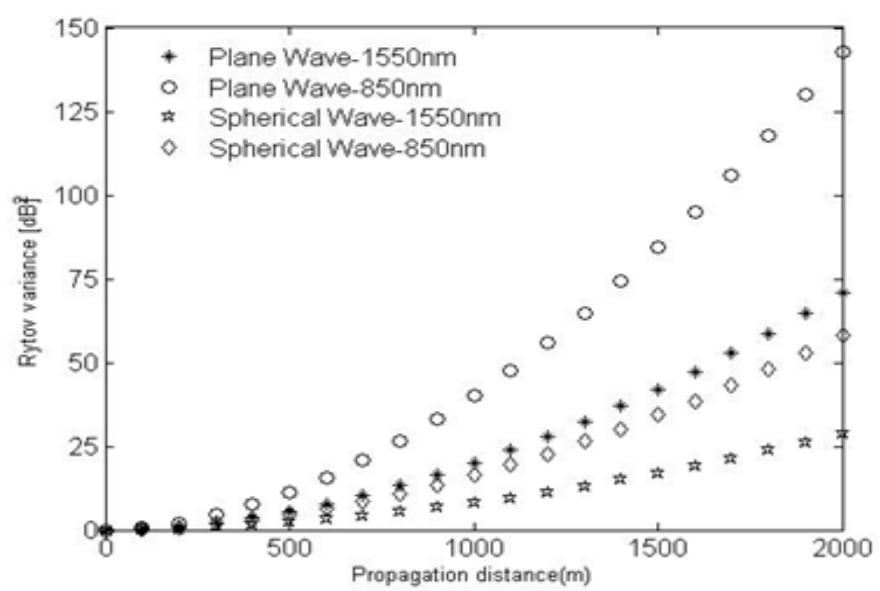

Fig. 4.Comparison of attenuation of the Relative variance for $C_{n}^{2}=10^{-12} \mathrm{~m}^{-23}$ 
The turbulence attenuation calculated depended on Rytov approximation and Andrews's method. The calculation proceeded with the following parameters: $\lambda=805 \mathrm{~nm}, 1550 \mathrm{~nm}, D_{\mathrm{REC}}=10 \mathrm{~cm}, \mathrm{~L}=(0-2000) \mathrm{m}$. The comparison of Rytov approximation and Andrews's method has shown in fig. (5).

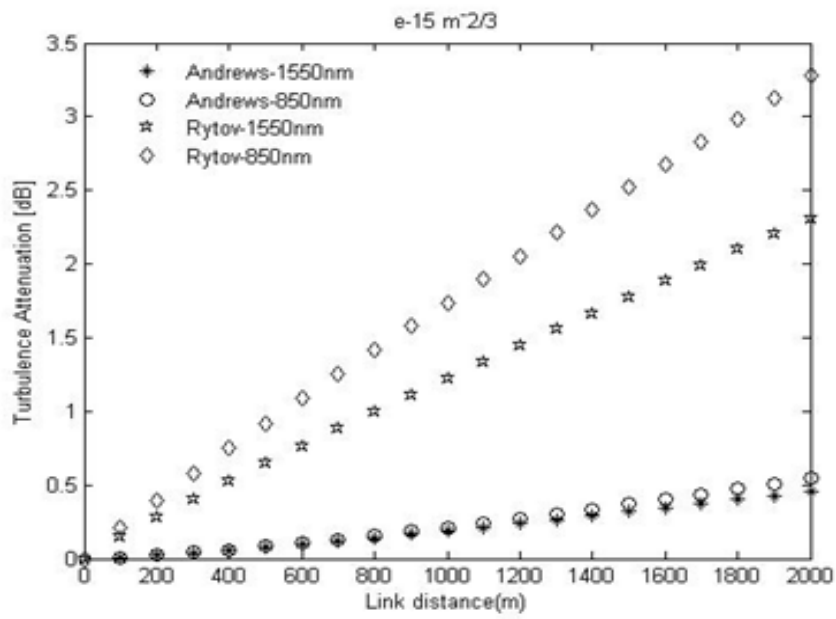

Fig. 5 Comparison of turbulence attenuation given by the Rytov approximation and Andrews's Method for $C_{n}^{2}=10^{-1 s} \mathrm{~m}^{-23}$.

We performed the same calculations also for $C_{n}^{2}=10^{-14} \mathrm{~m}^{-2 / 3}$. There is almost difference in the characteristics determined when the turbulence in increasing as shown in fig. (6)

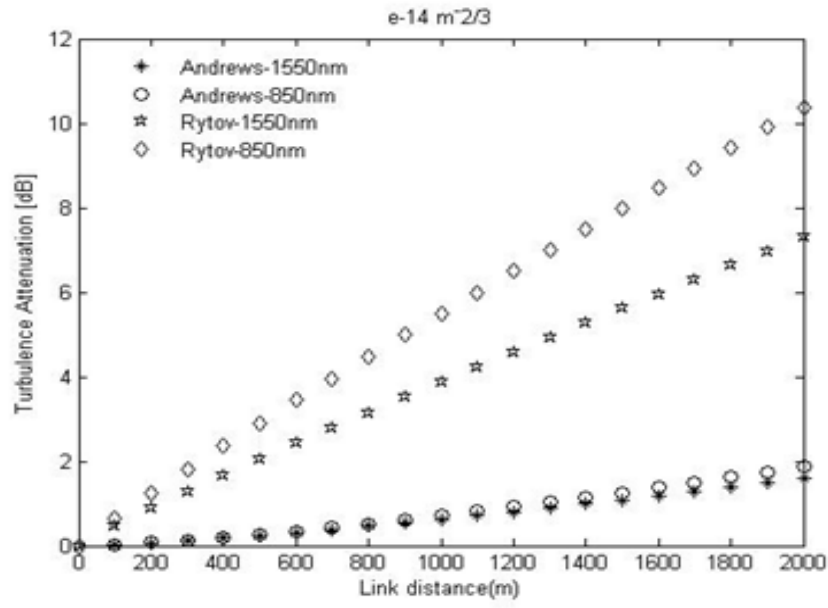

Fig. 6 Comparison of turbulence attenuation given by the Rytov approximation and Andrews's Method for $C_{n}^{2}=10^{-14} \mathrm{~m}^{-23}$.

Also the analogous calculations when the turbulence is strong $C_{n}^{2}=10^{-13} \mathrm{~m}^{-2 / 3}, C_{n}^{2}=10^{-12} \mathrm{~m}^{-2 / 3}$ as shown in fig. $(7,8)$. It is evident that the results from Andrews's method has no difference for $850 \mathrm{~nm}, 1550 \mathrm{~nm}$, on the other hand the results the turbulence has almost difference for the Rytov approximation and Andrews's method, this difference increased with increasing turbulence attenuation 


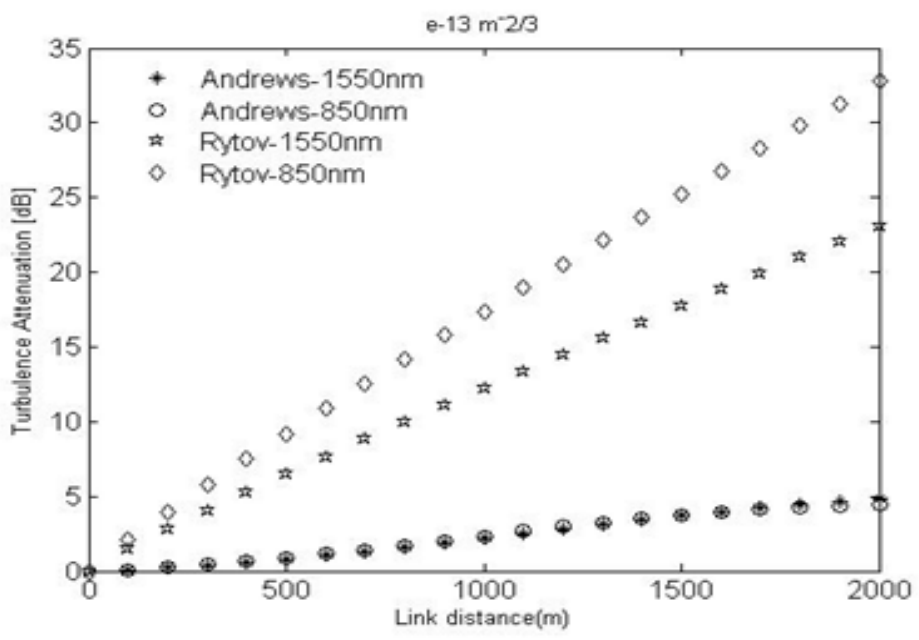

Fig. 7 Comparison of turbulence attenuation given by the Rytov approximation and Andrews's

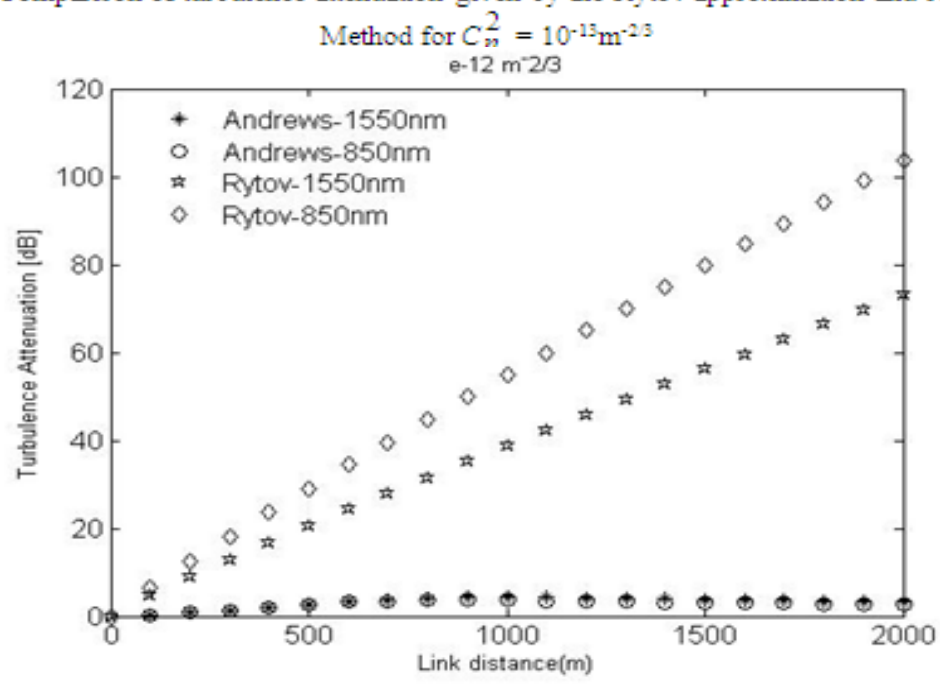

Fig. 8 Comparison of turbulence attenuation given by the Rytov approximation and Andrews's Method for $C_{n}^{2}=10^{-12} \mathrm{~m}^{-23}$

When the turbulence is not weak $\left(C_{n}^{2}=10^{-14} \mathrm{~m}^{-2 / 3}, C_{n}^{2}=10^{-13} \mathrm{~m}^{-2 / 3}, C_{n}^{2}=10^{-12} \mathrm{~m}^{-2 / 3}\right)$, the SNR calculated by using Tailor series for different values of $\alpha$ (the strength of turbulence). The SNR proceeded for $850 \mathrm{~nm}$ as shown in fig. (9), we observe that a minor difference between the curves of the SNR which has one type of turbulence such as $C_{n}^{2}=10^{-14} \mathrm{~m}^{-2 / 3}$

The same calculations run for wavelength $1550 \mathrm{~nm}$ fig. (10). There is almost no difference in the behavior of the curves and the SNR for many values of $\alpha$. 


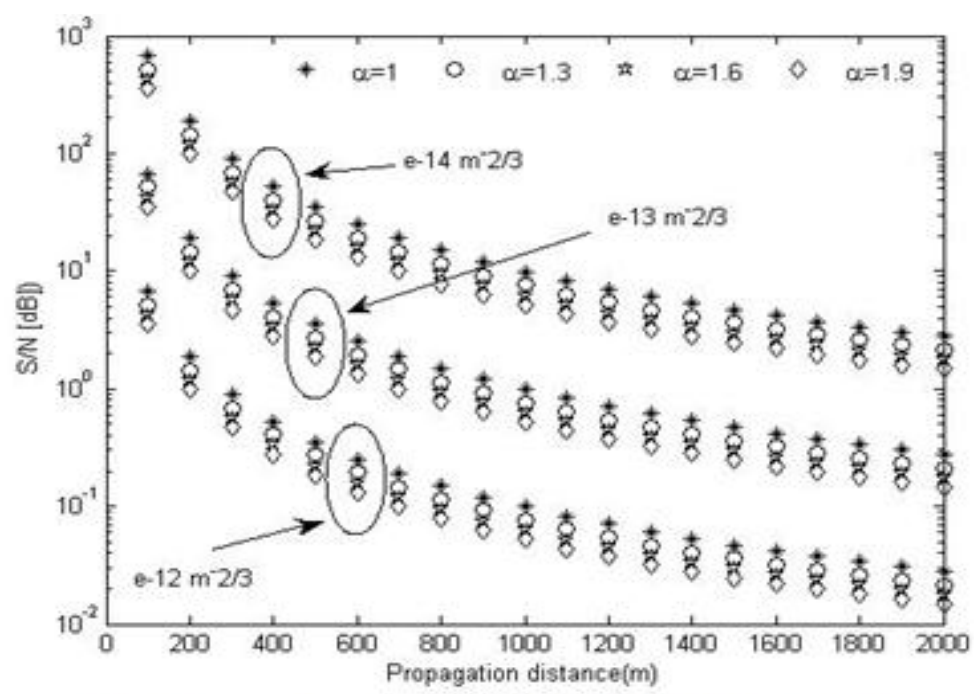

Fig. 9 Log scale of SNR at $850 \mathrm{~nm}$ for different values of $C_{n}^{2}$

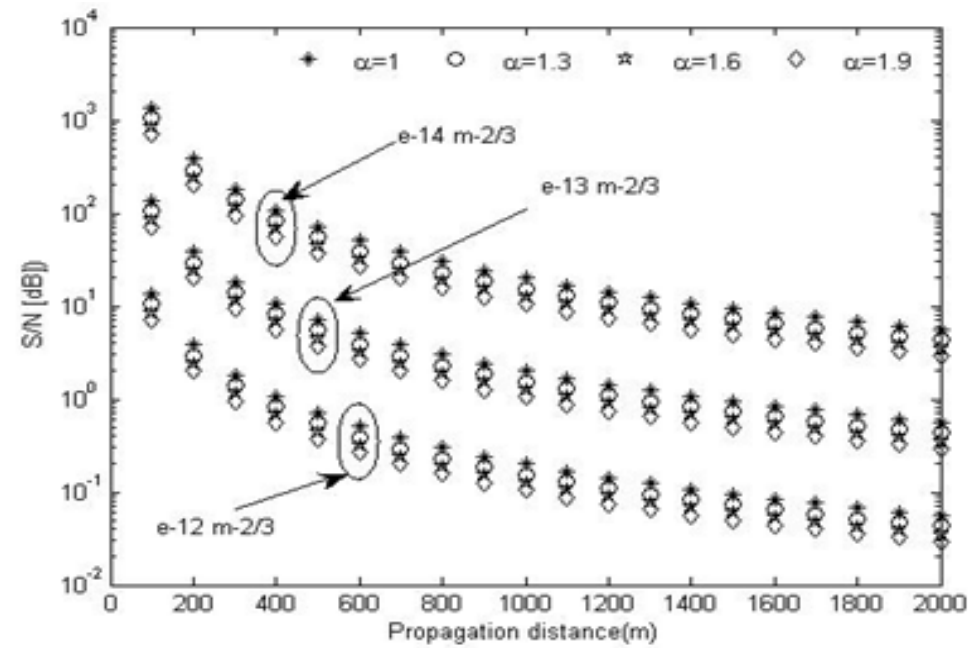

Fig. $10 \mathrm{Log}$ scale of SNR at $1550 \mathrm{~nm}$ for different values of $C_{n}^{2}$

\section{Conclusion}

The effect of atmospheric turbulence on refractive index variation has been analyzed. The spherical waves has less Rytov variance then plane waves, especially the wavelength $1550 \mathrm{~nm}$ which was less Rytov variance then $850 \mathrm{~nm}$ at different atmospheric turbulence applied. On the other hand the turbulence attenuation calculated using Andrews's method and Rytov approximation. In the case of calculating turbulence attenuation by Andrews's method we found that there is no difference between the attenuations of these wavelengths. Also calculated SNR when the turbulence is not weak, we employed the strength of turbulence $\alpha$ in this study. The wavelength $1550 \mathrm{~nm}$ has the best SNR compared with the wavelength $850 \mathrm{~nm}$ at the same strength of turbulence $\alpha$ and refractive index structure parameter $C_{n}^{2}$.

\section{References}

[1]. A. Prokeš, Modeling of Atmospheric Turbulence Effect on Terrestrial WOC Link, Radio Engineering, vol. 18, no. 1, 2008.

[2]. A. C. Motlagh, V. Ahmadi, Z. Ghassemlooy, K. Abedi, The Effect of Atmospheric Turbulence on the performance of the Free Space Optical Communications, International Symposium on Communication Systems, Networks and Digital Signal Processing, $6^{\text {th }}$, CNSDSP, 2008.

[3]. R. I. Roberto, S. M. IDRUS, and S. Ziran, Optical wireless communications: IR for wireless connectivity, New York: CRC Press, 2007.

[4]. P. Liu,K. Kazaura, K, Wakamori, M. Matsumoto, Studies on $C_{n}^{2}$ and its effects on free space optical communication system,8th Asia-Pacific Symposium on Information and Telecommunication Technologies, APSITT, 2010. 
[5]. L. Dordova, O. Wilfert, Calculation and Comparison of Turbulence Attenuation by Different Methods, Radio Engineering, vol. 19, no. $1,2010$.

[6]. L. C. Andrews, Field Guide to Atmospheric Optics, Washington: SPIE Press, 2004.

[7]. L. C. Andrews, Laser Beam Scintillation with Applications, SPIE Optical Engineering Press, Bellingham, Washington, 2001.

[8]. M. AL-Naboulsi, H, Sizum, F. De, Forne, Propagation of Optical and Infrared Waves in the Atmosphere, in the proceedings of the XXVIIIth URSI General Assembly in New Delhi, 2005, New Delhi (India): URSI.

[9]. X. M. Zhu, M. Khan, Free-Space Optical Communication through Atmospheric Turbulence Channels, IEEE Transactions on Communications, vol.50, no. 8,2002.

[10]. H. E. Nistazakis,T. A. Tsiftsis, G. S. Tombras, Performance analysis of free-space optical communication systems over atmospheric turbulence channels, IET Communication, vol. 3, Issue 8, pp. 1402-1409, 2009.

[11]. X. Guoliang, Z. Xuping, W. Junwei, F. Xiaoyong, Influence of atmospheric turbulence on FSO link performance, Proc. SPIE, Optical Transmission, switching, and Subsystems, vol. 5281, 2004. 